\title{
Global Connectivity of Prefrontal Cortex Predicts Cognitive Control and Intelligence
}

\author{
Michael W. Cole, ${ }^{1}$ Tal Yarkoni, ${ }^{2}$ Grega Repovs, ${ }^{3}$ Alan Anticevic, ${ }^{4}$ and Todd S. Braver ${ }^{1}$ \\ ${ }^{1}$ Psychology Department, Washington University, St. Louis, Missouri 63130, ${ }^{2}$ Institute of Cognitive Science, University of Colorado Boulder, UCB 345 , \\ Boulder, Colorado 80309, ${ }^{3}$ Department of Psychology, University of Ljubljana, Ljubljana, Slovenia, and ${ }^{4}$ Department of Psychiatry, Yale University School \\ of Medicine, New Haven, Connecticut 06519
}

Control of thought and behavior is fundamental to human intelligence. Evidence suggests a frontoparietal brain network implements such cognitive control across diverse contexts. We identify a mechanism — global connectivity-by which components of this network might coordinate control of other networks. A lateral prefrontal cortex (LPFC) region's activity was found to predict performance in a high control demand working memory task and also to exhibit high global connectivity. Critically, global connectivity in this LPFC region, involving connections both within and outside the frontoparietal network, showed a highly selective relationship with individual differences in fluid intelligence. These findings suggest LPFC is a global hub with a brainwide influence that facilitates the ability to implement control processes central to human intelligence.

\section{Introduction}

Solving the problems of everyday life requires effective control of thought and behavior. This is most evident when such cognitive control breaks down. For instance, individuals with brain lesions in the lateral prefrontal cortex (LPFC) have difficulty with common tasks such as planning and executing grocery shopping trips (Shallice and Burgess, 1991). Even healthy individuals are limited in their capacity for cognitive control, as measured by indices such as working memory capacity and general fluid (gF) intelligence (Conway et al., 2002). This "cognitive control capacity" appears to be highly general and life relevant, predicting important outcomes such as academic and vocational success (Engle et al., 1999; Gottfredson and Saklofske, 2009).

Recent evidence suggests cognitive control capacity may be supported by whole-brain network properties. For instance, van den Heuvel et al. (2009b) found that individuals with higher intelligence had more efficient whole-brain network organization. More recently, we found that a specific frontoparietal brain network thought to underlie cognitive control capacity (Cole and Schneider, 2007; Duncan, 2010) has especially high global connectivity (Cole et al., 2010b). In contrast to the whole-brain network property account (and accounts that emphasize specific connections among regions), this suggests global connectivity of specific control regions may be important for cognitive control capacity. This would allow for a mechanism by which specific

\footnotetext{
Received February 3, 2012; revised April 18, 2012; accepted May 12, 2012.

Author contributions: M.W.C., T.Y., G.R., and T.S.B. designed research; M.W.C. and T.Y. analyzed data; M.W.C., A.A., and T.S.B. wrote the paper.

This research work was supported by National Institutes of Health Grants MH66088, NR012081, MH66078, and MH66078-06A1W1. We thank Patryk Laurent and Jeremy Reynolds for their helpful feedback and Jordan Livingston, Lauren Ness, and Nicholas Fazzio for their help with data analysis.

Correspondence should be addressed to Michael W. Cole, Washington University, One Brookings Drive, Campus Box 1125, St. Louis, M0 63130. E-mail: mwcole@mwcole.net.

DOI:10.1523/JNEUROSCI.0536-12.2012

Copyright $\odot 2012$ the authors $\quad 0270-6474 / 12 / 328988-12 \$ 15.00 / 0$
}

control regions can access and influence other relevant networks (such as sensory-motor networks involved in task-relevant processing) to adaptively monitor and regulate ongoing behavior (Dehaene et al., 1998; Miller and Cohen, 2001).

In the current study we used a recently developed graph theoretic approach to neuroimage data, sometimes referred to as global brain connectivity (GBC) or weighted degree centrality (Cole et al., 2010b, 2011; Rubinov and Sporns, 2011). This approach enabled consideration and characterization of specific regions' full range of connectivity, including connections outside the canonical frontoparietal control network, allowing us to comprehensively examine the role of each region's global connectivity in human cognitive control and intelligence.

We tested our hypothesis that cognitive control capacity is supported by the global connectivity of specific control network regions using a convergent approach involving analyses of both task-related and resting state functional MRI data. First, we identified regions meeting stringent criteria for involvement in cognitive control using the N-back task, a benchmark probe of working memory and cognitive control (Braver et al., 1997). Second, we used resting state functional MRI data to test whether these regions had high GBC relative to the rest of the brain. Finally, we determined whether the GBC in any of these regions predicted individuals' intelligence and cognitive control abilities. This final test is critical because a demonstration that a given brain region has both reliable involvement in control and high GBC does not necessitate that the global connectivity of such a region has a functional role in supporting cognitive control capacity. Indeed, we found that the GBC of most regions meeting these criteria could not reliably predict intelligence, as the GBC of only a single region-left LPFC - could do so. This suggests that there may be a specific brain region that uses brainwide connectivity as a central mechanism for supporting cognitive control and intelligence. 


\section{Materials and Methods}

Participants. The data presented here were collected as part of a larger, multifaceted study. Individuals participated in 3 separate sessions, spaced a few days to a few weeks apart, to complete personality tests, mood questionnaires, neuropsychological tests, and the N-back fMRI scanner task. The first session was $3 \mathrm{~h}$ long and involved answering several standard paper-and-pencil questionnaires; the second was $2 \mathrm{~h}$ long and involved answering computerized questionnaires and cognitive tasks; the third was $2.5 \mathrm{~h}$ long, and involved an fMRI scan of the N-back task. The Raven Advanced Progressive Matrices, Set II (Raven et al., 1998) and The Cattell Culture Fair Test (Cattell et al., 1973) were included in the first session as measures of $\mathrm{gF}$. The standard scoring procedures for each test were used to compute measures from these tests. Data from these participants have been used in other articles to address questions distinct from those in the current study (Fales et al., 2008; Shamosh et al., 2008; DeYoung et al., 2009, 2010; Burgess et al., 2011).

One hundred twenty-one participants ( 70 female; mean age, 23 years; range, $18-40$ years) were recruited from the undergraduate population at Washington University $(n=60)$ or surrounding communities and received financial remuneration for their participation. The experiment was approved by the Washington University Institutional Review Board.

Participants were eliminated from N-back task-based analyses if they showed unusually poor performance, as indicated by multivariate analysis (Mahalanobis distance; indexed across six variables that coded the proportion of incorrect responses and response omissions for each type of trial). Five participants were eliminated on the basis of this criterion (Mahalanobis distance of $>22.46$, reflecting the corresponding $\chi^{2}$ value at $p<0.001$, with 6 degrees of freedom). A further 13 participants were eliminated due to at least one incomplete or missing BOLD scan or due to computer failure resulting in a loss of behavioral data. Finally, because the task analysis required that at least one error be committed on each type of 3-back trial, 10 participants who performed perfectly on novel trials were excluded. All results in the task analyses are therefore reported for 93 participants.

For the resting state functional connectivity analyses, 13 participants were removed from analysis because they had one or more incomplete or missing BOLD scans. Further, because a FreeSurfer gray matter mask was used for computing GBC for each participant, 14 participants with poor quality anatomical images (and resulting poor quality gray matter masks) were removed. We included 94 participants for the reported connectivity analyses. The different exclusion criteria for task-based and rest-based analyses resulted in 78 participants overlapping across analyses. The lack of complete overlap in the 2 samples might be considered statistically beneficial, as this increased statistical independence between the task-based activity and the rest-based connectivity results. In-house software was used for task activation analyses, while AFNI (Cox, 1996) and R (R Development Core Team, 2009) were used for connectivity analyses. In the sample of 94 participants used for most of the analyses, the mean age was 22 years (SD, 4.7 years; range, $18-39$ years).

The 3-back task. The 3-back task was administered using PsyScope (Cohen et al., 1993) on a Macintosh G3 (Apple Computer). Participants performed a standard version of the task that has been used in several previous studies of working memory (Gray and Braver, 2002; Gray et al., 2002, 2003, 2005; Schaefer et al., 2006; Yarkoni et al., 2009). Stimuli (either words or faces) were displayed sequentially on a screen. Participants were instructed to respond with the right index finger if the current stimulus matched the stimulus presented 3 trials back (target response) and with their right middle finger if the current stimulus was different (nontarget response). The 3-back trials were classified into 3 types: novel, target, and lure. Each participant's behavioral performance was assessed by computing their mean accuracy rate separately for each trial type.

On Novel trials ( $52 \%$ of all trials), the presented item was completely novel to the participant and had not been previously presented. On Target trials (32\%), the presented item was the same as the one presented 3 trials previously. On Lure trials (16\%), the current item had been presented at least once before but had not been presented exactly 3 trials back. The tension between targets and lures presented the main source of difficulty in the task. In theory, the inclusion of lure trials should prevent participants from simply using a recognition-based strategy and classifying any previously seen item as a target. In practice, however, participants performing the N-back often use a heuristic approach that allows for a combination of familiarity-based and control-based responding (Kane et al., 2007), implying that the results of many N-back studies may reflect the aggregate action of several very different cognitive processes.

For a given scanning run, stimuli were either all faces (unfamiliar attractive males and females intermixed, either neutral or smiling) or all words (concrete English nouns with neutral content, such as rooster, elbow, steel, fence, ball, tooth). These stimuli were used in a number of previous studies (Braver et al., 2001; Gray et al., 2002, 2003). Each stimulus was presented individually for $2.0 \mathrm{~s}$, with a fixation point (crosshair) shown between stimuli. The task was presented in 6 scanning runs, each consisting of 2 blocks of 32 task trials randomly interspersed with 18 variable intertrial intervals included to facilitate deconvolution of eventrelated responses. Task blocks were also preceded and followed by $15-$ frame blocks of resting fixation, for a total of 45 fixation frames per run.

fMRI data acquisition and preprocessing. Whole-brain images were acquired on a 3T Allegra System (Siemens). Structural images were acquired using a magnetization-prepared rapid gradient-echo $\mathrm{T} 1$-weighted sequence. Functional images were acquired using an asymmetric spin-echo echoplanar sequence sensitive to BOLD magnetic susceptibility ( $\mathrm{TR}=2360$ $\mathrm{ms}, \mathrm{TE}=50 \mathrm{~ms}$, flip $=90^{\circ}$ ). Each scanning run gave 149 sets of brain volumes (32 contiguous, $4 \mathrm{~mm}$ thick axial images, $4 \times 4 \mathrm{~mm}$ in-plane resolution).

Preprocessing was conducted separately for the general linear model (GLM) and functional connectivity (fcMRI) analyses. For the GLM analysis, functional images were corrected for movement, normalized within each scanning run, and temporally aligned within each brain volume. Next, images were transformed into atlas space (Talairach and Tournoux, 1988) and smoothed with a Gaussian filter (6 mm FWHM) before statistical analysis.

For the resting state fcMRI analysis, functional images were extracted from the rest periods between task blocks using a previously validated method (Fair et al., 2007). Although there are some criticisms of using signal between task blocks as rest (Barnes et al., 2009), we minimized these concerns in the following way (see Discussion for further consideration of this issue): we used rest images that were $14.16 \mathrm{~s}$ ( 6 frames) or more past the end of every task block and $4.72 \mathrm{~s}$ ( 2 frames) or fewer into the start of every task block. Further, mean signal amplitude for each rest block was subtracted for each voxel before rest block concatenation. There were 3 rest blocks per run, resulting in 35 rest frames per run (after removing the first 5 frames of each run). There were $8.26 \mathrm{~min}$ of rest total (across the 6 runs) per participant.

These resting state functional images were further preprocessed with the following steps: (1) slice-time correction, (2) removal of first 5 images from each run to reach steady state, (3) rigid body motion correction, (4) bandpass filtering $(0.009<f(t)<0.08)$, (5) removal of a set of nuisance regressors including signals from the ventricles, deep white matter, whole brain average, motion correction parameters, and first derivatives of these regressors, (6) additional removal of local white matter (using 16 $\mathrm{mm}$ radii spheres) nuisance regressors on a voxelwise basis (ANATICOR) (Jo et al., 2010), and (7) smoothing with a $6 \mathrm{~mm}$ spherical dilation of gray matter voxels (to avoid averaging non-brain voxel noise with the gray matter). These steps helped to ensure that spurious signal confounds did not impact our results. Finally, following GBC processing but before group analysis, the images were transformed into atlas space (Talairach and Tournoux, 1988).

fMRI GLM Analysis. The regions of interest (ROIs) were identified using a GLM data analysis approach (Friston et al., 1995). Because the experiment was designed for a mixed blocked/event-related analysis (Visscher et al., 2003; Dosenbach et al., 2006), separate regressors were used to estimate sustained (i.e., blocked) and transient (i.e., eventrelated) activation. Sustained activation during task blocks relative to fixation periods was modeled with an assumed response shape by convolving a boxcar with a model hemodynamic response function. Eventrelated effects were estimated empirically using a basis set of finite impulse response regressors. Each event-related effect was modeled using a set of 7 staggered regressors spanning a total epoch duration of $16.52 \mathrm{~s}$. 
All analyses were conducted on summary magnitude estimates obtained by averaging the signal at the third, fourth, and fifth time points of each time course (i.e., the expected peak of activation).

Six different event-related effects of interest were modeled in the GLM, representing separate coding of correct and incorrect responses for each of the 3 different trial types (e.g., correct novel trials, incorrect novel trials, correct target trials). This approach enabled us to analyze the data using a $3 \times 2$ repeated-measures ANOVA with trial type and accuracy as factors. In addition to the 6 effects of interest, each GLM contained a number of covariates of no interest, including 6 covariates coding for experimental condition at the block level (reflecting a $2 \times 3$ factorial combination of stimulus type and mood induction valence), a parametric covariate coding for trial-by-trial differences in reaction time to control for time-on-task effects (Yarkoni et al., 2009), a covariate coding all trials with omitted response separately [mean, 3.1\% (SD, 3.2\%) of all trials]), onset and offset covariates that modeled the initiation and termination of each block of trials to control for reliable task-independent activation that occurs at the start and end of task blocks (Dosenbach et al., 2006), and nuisance regressors modeling separate intercepts and linear trends for each BOLD run.

For all within-subject analyses, brain regions were identified using whole-brain paired $t$ tests that compared activation at each voxel between conditions of interest. For individual differences in N-back performance, we conducted a whole-brain correlation analysis between mean response accuracy and task-related activation. Because individual differences analyses typically have substantially lower power to detect effects than within-subject analyses (Braver et al., 2010; Yarkoni et al., 2010), we sought to improve signal-to-noise by averaging both response accuracy and brain activation across all trial types (i.e., Novel, Target, and Lure) before analysis.

Statistical maps were corrected for multiple comparisons using a whole-brain false discovery rate (FDR) (Nichols and Hayasaka, 2003) threshold of 0.05 and a clusterwise (extent) threshold of $\geq 9$ contiguous statistically significant voxels. For the subsequent conjunction analysis that sought to identify regions common to multiple contrasts, all maps included in each conjunction were individually corrected for multiple comparisons before masking (Nichols et al., 2005).

For visualization purposes, statistical maps were mapped onto a 3-dimensional representation of the cortical surface (the PopulationAverage, Landmark- and Surface-Based atlas; Van Essen, 2005) using Caret software (Van Essen et al., 2001; http://brainvis.wustl.edu/wiki/index. php/Caret:About).

$G B C$ analyses. A region's GBC was computed as the average connectivity of that region with the rest of the brain (equivalent to unthresholded weighted degree centrality) (Cole et al., 2010b). Several studies have used unthresholded weighted degree centrality in the past as well (Cole and Schneider, 2007; Langer et al., 2011; Rubinov and Sporns, 2011). Analyses were restricted to each subject's individual gray matter mask (dilated by one functional voxel to accommodate spatial smoothing of functional data), obtained using FreeSurfer (Fischl et al., 2002). The brainwide GBC analyses were performed according to the following steps for each volume of interest (either a region or a voxel): First, a correlation was obtained with each voxel's resting state time series (excluding itself). Second, the correlations were transformed to Fisher Z $(\mathrm{Fz})$ values. Third, all (positive, negative, or absolute value) correlations were averaged to produce a GBC value. Voxelwise GBC maps were computed using AFNI's 3dTcorrMap, while GBC with an ROI was computed using custom scripts running AFNI's 3dDeconvolve, 3dcalc, and 3 dmaskave (Cox, 1996). For the ROI analyses, connectivity was based on correlations with the average of the time series from all within-region voxels.

Importantly, we never averaged positive and negative connections, given that mixed positive and negative values could cancel each other (e.g., averaging 0.2 and -0.2). Instead, for analyses using positive connections, each subject's negative connections were excluded. Similarly, for analyses using negative connections, each subject's positive connections were excluded. All voxels were included for analyses using absolute values. Note that the main GBC analysis (see Fig. 2) used positive connections only, given the current controversy regarding negative connections (Fox et al., 2009; Murphy et al., 2009), though the LPFC ROI effects were also significant for the negative-only and absolute value analyses.

The "top 10\% GBC" analyses were conducted by identifying the voxels with GBC $>90 \%$ of the gray matter voxels (Cole et al., 2010b; Lynall et al., 2010; Fornito et al., 2011). Top percentages were calculated as the number of voxels with higher GBC than the region divided by the total number of voxels in the brain.

For individual differences correlations, each volume's (either a region's or a voxel's) GBC was extracted for each subject, and a correlation was computed based on individual variability in this measure and in the composite gF behavioral measure (using AFNI's 3dRegAna). For the whole-brain GBC analyses, the statistical map was thresholded at $p<$ 0.01 uncorrected, then corrected for multiple comparisons (familywise error) to $p<0.05$ using a cluster threshold. AFNI's AlphaSim (Cox, 1996) was used to compute a cluster size threshold of 16 voxels based on 10,000 Monte Carlo simulations. These simulations used a group gray matter mask (gray matter mask voxels with overlap across at least $50 \%$ of subjects), and image smoothness for the simulations was estimated using AFNI's 3dFWHMx.

Restricted GBC analyses. We assessed the origin of the LPFC connectivity-gF correlation by recalculating this correlation for each in a series of connectivity strength ranges. LPFC's group average connectivity map (excluding voxels within LPFC itself) was used to define the across-voxel strength ranges, starting at an $\mathrm{Fz}$ of 0 and going up and down in increments of 0.05 until only a small number of voxels remained. Note that these are subsets of the full absolute value LPFC GBC-gF analysis ( $p=$ 0.002 ), such that multiple comparisons are controlled for using the logic of the protected Fisher's least significant difference approach. The same procedure used for calculating GBC was used here (i.e., calculating LPFC's time series correlation with each voxel, then averaging) but was restricted to each group average map strength range.

The strength range analysis indicated that LPFC's connectivity could be roughly separated into 3 broad functional systems based on connectivity strengths. The cognitive control network had strong positive connectivity $(\mathrm{Fz}>0.2)$, the default mode network had relatively strong negative connectivity $(\mathrm{Fz}<-0.05)$, and sensory-motor networks had relatively weak positive and negative connectivity $(-0.15<\mathrm{Fz}<0.2)$. We used these group average strength ranges, along with independently identified voxel masks, to define the 3 systems.

The cognitive control and default mode networks were defined using NeuroSynth (Yarkoni et al., 2011), which identifies locations with a high probability of their being reported in studies using specific terms. The cognitive control network locations are based on 93 studies (forward inference) using the term cognitive control. The default mode network locations are based on 77 studies using the term "default mode." Locations for both cognitive control and default mode networks were downloaded August 14, 2011, with $p<0.05$ thresholds and FDR corrections for multiple comparisons. The sensory-motor locations were based on probabilistic cytoarchitecture obtained using AFNI TT_N27_CA_EZ_PMaps, which is derived from the SPM Anatomy toolbox (Eickhoff et al., 2005). All voxels in the primary visual, primary auditory, primary motor, and somatosensory cortices were included. Any voxels that overlapped between any 2 of the 4 system masks were excluded from the analysis.

The LPFC connectivity-gF correlations were computed for each functional system separately. To better ensure separation of the systems, the group average strength ranges indicated above were used to restrict the voxels contributing to each system's average LPFC connectivity. In addition, only positive connections were used (selected separately for each subject) for control network voxels and only negative connections were used for default mode network voxels, based on previous activationbased and connectivity-based studies demonstrating anticorrelations between these systems (Fox et al., 2005). The absolute value of each connection was taken before averaging for the sensory-motor networks to avoid averaging of positive and negative connections (which could cancel out individual difference effects). 

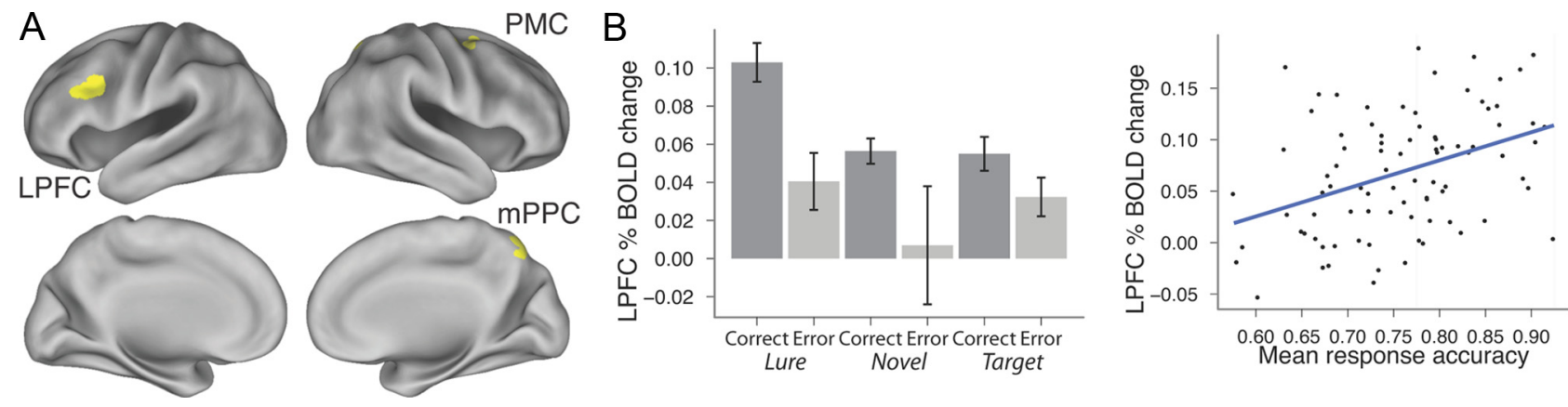

Figure 1. Cognitive control regions, as defined by successful cognitive control. $A$, Rols were defined based on brain activity during successful N-back task performance. The following highly selective criteria were used: preferential activation for trials requiring flexible control (lures), correct $>$ incorrect trials, positive correlation with accuracy across participants. All 3 of these regions were hubs (in top 10\% connectivity in the brain). $\boldsymbol{B}$, Illustration of effects defining the LPFC ROI. LPFC was more active across correct relative to error trials and more active for lure than other trial types. LPFC activity (correct trials across all trial types) was also correlated with overall response accuracy across individuals $(r=0.39, p=0.0002)$.

Table 1. Regions identified in the task-based and connectivity analyses

\begin{tabular}{llrrllll}
\hline & Volume $\left(4 \mathrm{~mm}^{3}\right)$ & \multicolumn{1}{c}{} & \multicolumn{1}{c}{ L } & $Z$ & Lure versus others & Correct versus error & Accuracy correlation \\
\hline $\operatorname{LPFC}^{a}$ & 17 & -43.4 & 13.6 & 29.4 & $F=10, p=0.002$ & $F=11, p=0.001$ & $r=0.39, p=0.0002$ \\
$\mathrm{mPPC}^{a}$ & 17 & 11.4 & -64.3 & 52.2 & $F=11, p=0.001$ & $F=6, p=0.02$ & $r=0.42, p=0.0001$ \\
$\mathrm{PMC}^{a}$ & 11 & 28.5 & -4.6 & 54.2 & $F=9, p=0.003$ & $F=15, p=0.0002$ & $r=0.29, p=0.007$ \\
$\operatorname{LPFC}^{b}$ & 16 & -46.3 & 14.9 & 28.5 & & & \\
$\operatorname{LPFC}^{c}$ & 9 & -44.2 & 13.7 & 29.8 & & & \\
\hline
\end{tabular}

${ }^{a}$ Regions identified in the task-based analysis.

${ }^{b}$ Regions identified in the voxelwise GBC-gF correlation (connectivity) analysis.

'Overlap among task-based, connectivity, and Burgess et al. (2011) analyses.

\section{Results}

Identifying core cognitive control regions

Brain regions were identified as important for cognitive control based on their activation profile in a group of 93 participants scanned during performance of the N-back task of working memory and executive control. First, these regions had to show increased activation for lure/interference trials, which had high control demands relative to other N-back trials (Gray et al., 2003). Second, they had to demonstrate increased activation on correct relative to error N-back trials, indicating a trial-by-trial contribution to successful performance. Last, average N-back trial-related activity had to correlate with task accuracy across participants, indicating a role in performance-related individual differences. Three regions within the frontoparietal control network were identified that met these strict criteria: left LPFC, right premotor cortex (PMC), and right medial posterior parietal cortex (mPPC) (Fig. 1, Table 1).

\section{Identifying cognitive control regions with high GBC}

We next tested the hypothesis that these 3 regions might be central to cognitive control because they serve as global hubs-regions highly connected to many other brain regions, as indexed by the GBC measure. In previous work, we demonstrated that regions within the frontoparietal control network tend to have the highest GBC within the brain (Cole et al., 2010b). In the present study, GBC was assessed using standard resting state functional connectivity MRI (rs-fcMRI) measures from $\sim 8 \mathrm{~min}$ of resting state data acquired in each participant from interspersed rest blocks occurring between task blocks, an approach validated in previous work (Fair et al., 2007). Note that rs-fcMRI is widely viewed as a useful brain connectivity measure because it is correlated with known structural connectivity (Honey et al., 2009; van den Heuvel et al., 2009a), though it likely reflects "functional/Hebbian" connectivity as well (Lewis et al., 2009); is highly consistent with well-established functional networks (Smith et al., 2009; Van Dijk et al., 2010); likely reflects intrinsic connectivity as it is present even under anesthesia (Vincent et al., 2007); and is highly replicable (e.g., within individuals, between individuals, across scanners, across parameters) (Shehzad et al., 2009; Van Dijk et al., 2010). Each region's GBC was computed as its average positive rs-fcMRI connectivity with the rest of the brain. Based on previously defined criteria for what constitutes a hub region (Cole et al., 2010b), we found that all 3 regions could be considered global connectivity hubs, as their GBC was in the top $10 \%$ compared across the whole brain.

\section{Using GBC to predict cognitive control and intelligence}

We next tested for a relationship between these regions' GBC and cognitive control capacity. Importantly, $\mathrm{gF}$ was used rather than $\mathrm{N}$-back performance to assess cognitive control capacity with an independent and more robust measure of stable, traitlike, and domain-general individual differences. $\mathrm{gF}$ is a domain-general measure of the fluid aspects (those aspects independent of knowledge and culture) of general intelligence that has been used in psychometric research for over a century of studies involving thousands of participants (Gottfredson, 1997). Robust findings demonstrate its relevance to a wide variety of both psychometric and real-life tasks (Gottfredson, 2002), providing good reason to expect that results based on $\mathrm{gF}$ will generalize to a wide variety of other tasks, especially those in which cognitive control demands are high (Gottfredson, 1997). We quantified $\mathrm{gF}$ as the $\mathrm{z}$-normalized average of 2 well-established measures of gF collected outside the scanner: the Raven Advanced Progressive Matrices, Set II (Raven et al., 1998) and the Cattell Culture Fair Test (Cattell et al., 1973).

Individual differences correlations between positive-connectivity GBC (computed using positive connections only) and $\mathrm{gF}$ were statistically significant for LPFC $(r=0.32, p=0.0015)$ but not for mPPC $(r=0.08, p=0.42)$ or PMC $(r=0.13, p=$ $0.22)$ (Fig. 2A). This LPFC GBC-gF correlation remained statistically significant after controlling for the effect of age $(r=0.24$, 
$p=0.018)$. These results demonstrate that individuals with higher GBC in LPFC have greater cognitive control capacity, suggesting brainwide connectivity with a specific control region helps support cognitive control abilities. Further, these results converge with results from previous N-back studies (Gray et al., 2003; Burgess et al., 2011), based on both brain (resting state connectivity) and behavior (fluid intelligence) measures, to suggest that left LPFC is central to the implementation of cognitive control.

The main GBC analyses used positive connections only, given the current controversy regarding negative connections (Fox et al., 2009; Murphy et al., 2009). However, the LPFC region effects were also significant for the negative-only ( $r=$ $-0.29, p=0.004)$ and absolute value $(r=$ $0.31, p=0.002)$ analyses. This suggests that negative connectivity might be functionally relevant with regard to gF.

We also examined the predictive power of GBC with a second independent measure of cognitive control, working memory capacity, as indexed by the wellestablished operation span task (Conway and Engle, 1996). Convergent with the gF results, a significant relationship was only observed for GBC in LPFC: $r=0.24, p=$ 0.019 (for $\mathrm{MPPC}$ and PMC, $p>0.15$ ). In subsequent analyses, we focus primarily on $\mathrm{gF}$ given the well-established generality and life relevance of this cognitive construct (Gottfredson and Saklofske, 2009).

To assess specificity to fluid (as opposed to crystallized) intelligence, we determined whether LPFC GBC was also correlated with a measure of crystallized intelligence: the vocabulary subtest of the Wechsler Adult Intelligence Scale-Third Edition test (Wechsler, 2005). We found no significant correlation between crystallized intelligence and LPFC GBC ( $r=0.12, p=0.25)$, despite there being a significant correlation between crystallized intelligence and $\mathrm{gF}(r=0.43, p=$ 0.00001 ). Further, the LPFC GBC-gF correlation was (marginally) significantly higher than the LPFC GBC-crystallized intelligence correlation $(\mathrm{z}=1.87, p=0.06)$. These results suggest that the relationship between LPFC GBC and intelligence was specific to fluid aspects of intelligence, consistent with our interpretation that this relationship reflects cognitive control processes underlying intelligence.

\section{Assessing the relative specificity of the LPFC GBC-gF effect}

The primary goals of previous work investigating the neural basis of intelligence have been to identify whole-brain network contributions to intelligence (van den Heuvel et al., 2009b), regionspecific activity contributions to intelligence (Duncan, 2000), or connection-specific contributions to intelligence (Song et al., 2008). In contrast, due to our hypothesized mechanism of cognitive control, our primary goal was to identify how specific regions' global connectivity might contribute to intelligence. As a critical aspect of this goal, we next sought to assess the relative specificity of the observed LPFC GBC-gF effect. We did this by examining GBC-gF correlations separately in all brain voxels. Remarkably, only a single significant region was identified ( $p<0.05$, family-wise error corrected for multiple comparisons), despite the relatively large sample size, with its location closely overlapping the LPFC region identified in the task-based analysis (Fig. 2 B, Table 1B). This suggests the GBC-gF effect was specific to LPFC.

Still, it remains possible that there were effects just below the statistical threshold used in this analysis. To assess this possibility and further evaluate the robustness of the LPFC GBC-gF effect, we compared the LPFC GBC-gF correlation with the GBC-gF correlation of every voxel in the brain (Fig. $3 A$ ). We accomplished this using a standard approach for comparing dependent correlations (Meng et al., 1992). Supporting the specificity of LPFC, we found that much of the brain had a lower GBC-gF correlation $(p<0.05$, familywise error corrected for multiple comparisons).

We might expect, based on previous activity studies showing strong relationships with intelligence (Gray et al., 2003), that control network regions such as anterior PFC and anterior cingulate cortex would show strong GBC-gF correlations. Further supporting this possibility, these regions also have among the highest GBC in the brain (Cole et al., 2010b). Surprisingly, these 
A

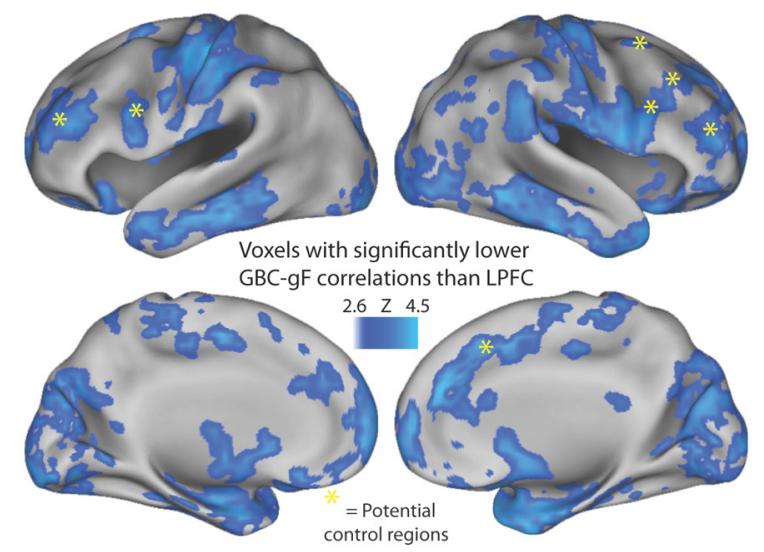

B

B
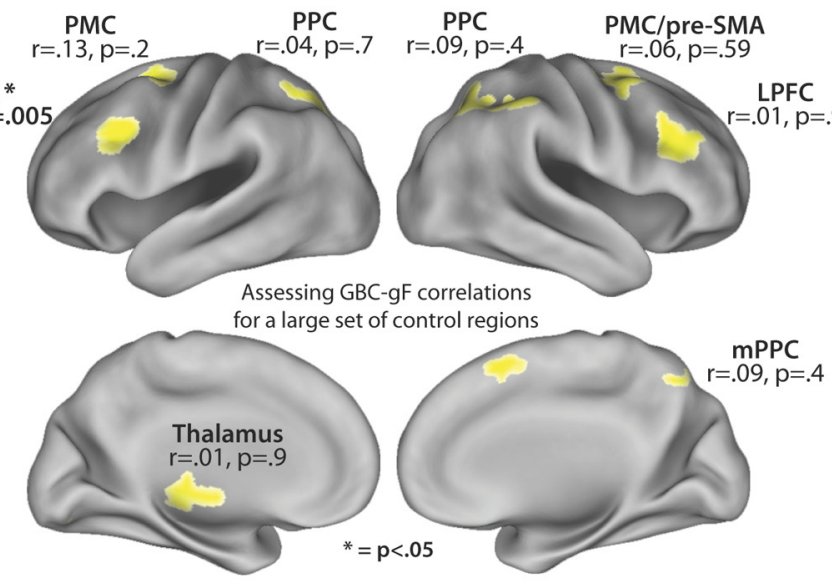

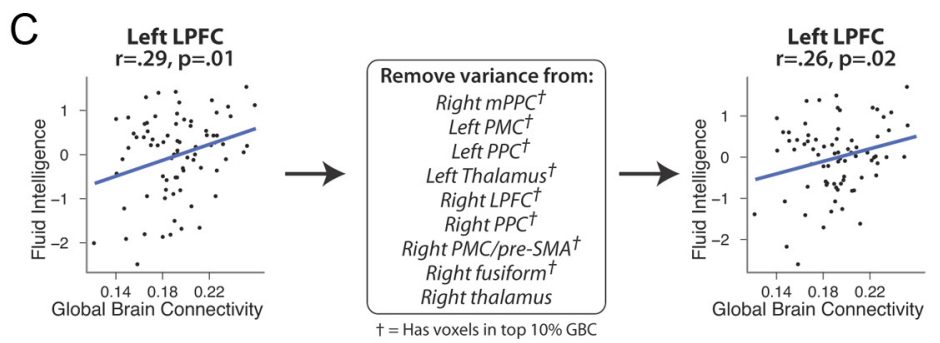

Figure 3. Examining the specificity of the left LPFC GBC $-\mathrm{gF}$ correlation. $A$, The LPFC GBC $-\mathrm{gF}$ correlation was compared with the GBC $-\mathrm{gF}$ correlation of every brain voxel. Significant voxel clusters are shown ( $p<0.05$, corrected for multiple comparisons). This supports the specificity of the LPFCGBC $-\mathrm{gF}$ effect and demonstrates that the contribution of global connectivity to intelligence is not uniform throughout the brain. Potential control regions (based on previous studies) are highlighted. $B$, The GBC $-\mathrm{gF}$ correlation was calculated for a larger set of regions from Burgess et al. (2011) that appeared likely to show GBC- $\mathrm{gF}$ correlations. Using the same dataset as here, Burgess et al. (2011) identified 10 regions (the largest 8 are depicted here) with N-back lure fMRI activity correlating with individual differences in cognitive control capacity (e.g.gF). Despite these regions' activities correlating with gF, only left LPFC (substantially overlapping with the previously defined LPFC region) showed a significant GBC - gF correlation. C, The left LPFC GBC $-\mathrm{gF}$ correlation was computed before and after removing the variance from all Burgess et al. (2011) regions (excluding left $L P F C$ ). The GBC $-\mathrm{gF}$ correlation for the left LPFC region (as defined in Fig. 1) was largely unaffected by removing the variance from the Burgess et al. (2011) regions. This demonstrates that the left LPFC GBC $-\mathrm{gF}$ correlation was statistically independent of a large set of cognitive control regions. Note that 9 of the 10 regions had voxels in the top $10 \% \mathrm{GBC}$, demonstrating that the $\mathrm{GBC}-\mathrm{gF}$ correlation is not guaranteed simply due to a control region having both gF-correlating activity and high GBC.

were among the many regions showing significantly smaller GBC-gF effects than LPFC (Fig. 3A, highlights).

It still remains possible that other control network regions, especially those with activity correlating with $\mathrm{gF}$, might also show significant GBC-gF correlations, reducing the specificity of the LPFC effect. We increased our ability to detect such correlations (relative to the previous voxelwise analysis) using an ROI approach with a larger set of control network regions, identified by Burgess et al. (2011) with the same large dataset as here (Fig. 3B). Burgess et al. (2011)identified these regions using criteria similar to (but less conservative than) our own (Fig. 1), but with one key difference: the activity in all 10 regions (the 3 we had identified, plus an additional 7) correlated with gF. We found that 9 of the 10 regions had voxels in the top $10 \%$ of GBC, further suggesting they might show GBC-gF correlations.

Nevertheless, we again found a remarkable degree of specificity in the GBC-gF effect, since the correlation was significant only in the left LPFC region $(r=0.29, p=0.005$ for left LPFC; $p>0.19$ for all other regions). Note that several regions had outlier subjects (GBC further than 2 SD from the group mean); however, the results were similar when including or excluding them. This left LPFC region overlapped with the LPFC regions identified in the first analysis (Fig. 1) and the whole-brain search analysis (Fig. 2 B), such that results from all 3 analyses overlapped in a common set of voxels (Table 1C). These results demonstrate that regions showing activity-gF correlations and high GBC are not guaranteed to show GBC-gF correlations. In other words, the results suggest $\mathrm{GBC}-\mathrm{gF}$ effects may be mechanistically dis- tinct from activity-gF effects (e.g., activity reflects within-region processing, while GBC reflects access to a wide variety of taskrelevant regions for implementing control).

It remains possible that the GBC-gF effect was actually distributed throughout the control network, and that, despite the highly statistically significant effect in left LPFC, the GBC metric was not sensitive enough to identify the effect in each region individually. We therefore averaged the GBC values across all the control regions (excluding left LPFC) to look for a network-wide effect and found that the resulting GBC-gF correlation was still not significant $(r=0.06, p=0.59)$. Moreover, this network-wide correlation was significantly lower than the left LPFC GBC-gF correlation $(\mathrm{z}=2.0, p=0.046)$.

Finally, we ruled out a network-wide effect using partial regression, testing whether the left LPFC GBC-gF correlation was statistically independent of the other 9 control regions (Fig. 3C). First, to remain conservative, subjects were removed from this analysis if they were outliers for any of the 10 regions (this removed 17 subjects), which reduced the LPFC GBC-gF correlation slightly $(r=0.29, p=0.01)$. Next, GBC values from each of the regions (excluding left LPFC) were included in a multiple regression model with the $\mathrm{gF}$ values. The residuals from this model (i.e., with gF-correlating individual differences variance from the 9 regions removed) were then correlated with the original left LPFC region's GBC values. The left LPFC GBC-gF correlation was almost identical and remained statistically significant $(r=0.26, p=0.03)$, suggesting that the left LPFC effect was statistically independent from the other regions. This effect re- 

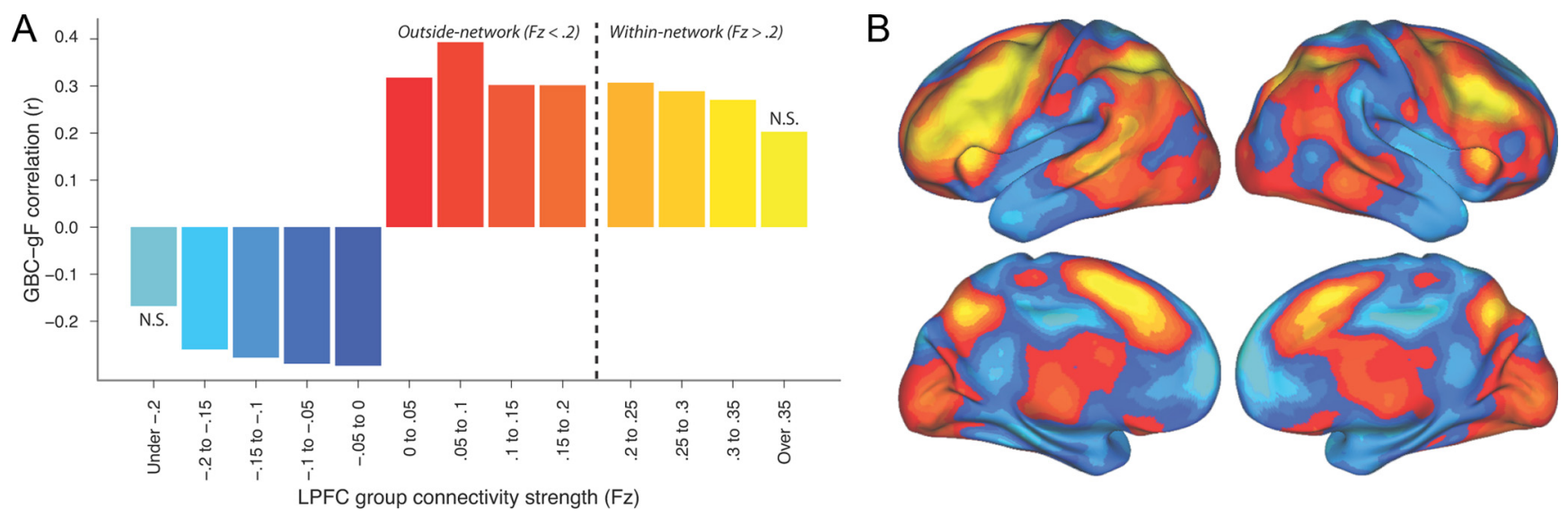

Figure 4. Identifying the origin of the $\mathrm{LPFC} G B C-\mathrm{gF}$ correlation. $A$, We systematically restricted the connectivity contributing to the calculation of the $G B C-g F$ correlation to determine the contributing connectivity strength ranges. LPFC's group average connectivity map was split into $\mathrm{Fz} 0.05$ "strength bands" and restricted GBC - $\mathrm{gF}$ correlations calculated using those voxels. All strength ranges were significant, except the highest and lowest, likely due to the low number of voxels included in these 2 strength ranges. This likely indicates that the $\mathrm{GBC}-\mathrm{gF}$ effect is global in origin, such that the more voxels that are included the better the connectivity - $\mathrm{gF}$ correlation. This is in contrast to the effect originating from high-strength connectivity, in which restricting to higher strength connections would improve the connectivity-gF correlation. $\boldsymbol{B}$, The LPFC seed connectivity group map is shown, colored by connectivity strength ranges (corresponding to colors in $A$ ).

mained even when restricting the 9 regions to the voxels in the top $10 \%$ GBC ( $r=0.25, p=0.04)$. Together, all these analyses further support the conclusion that the GBC-gF effect was highly selective to left LPFC and, moreover, was independent of other cognitive control regions despite their high $\mathrm{GBC}$ and gFcorrelating activity.

\section{Ruling out effects of motion}

A recent study by Power et al. (2012) established that even subtle movement can alter rs-fcMRI estimates, such that group or individual differences in motion might lead to false conclusions regarding differences in rs-fcMRI estimates. This appears unlikely to be able to explain the observed GBC-gF correlation, given its apparent specificity to LPFC (motion likely affects the entire brain, or at least more than just a single region). To remain conservative, however, we tested whether motion could explain the observed LPFC GBC-gF correlation.

First, we estimated overall motion [framewise displacement (FD)] as described by Power et al. (2012), regressed out each subject's motion from the gF scores, and found that the LPFC GBC-gF correlation was almost identical to that before $(r=0.30$, $p=0.004)$. We next used the "scrubbing" method recommended by Power et al. (2012) and found that the LPFC GBC-gF remained statistically significant $(r=0.27,0.0075)$ despite using a highly conservative threshold $[\mathrm{FD}=0.3, \mathrm{DVAR}=0.3$; Power et al. $(2012)$ used FD $=0.5, \mathrm{DVAR}=0.5)$ to remove a large amount of potentially motion-contaminated data. These results strongly suggest that the LPFC GBC-gF correlation is not an artifact of motion.

\section{Determining whether the left LPFC GBC-gF effect was global in origin}

We next sought to more thoroughly understand the source of this GBC-gF effect to determine whether it was truly global in nature or actually reflected a dominant effect of a subset of LPFC connections. LPFC connectivity was restricted to different ranges (both positive and negative) of connectivity strength, and we examined how these restrictions changed the GBC-gF correlation (Fig. 4). Strength ranges were specified starting at an $\mathrm{Fz}$ of 0 and going up and down in 0.05 bins. We found that the correlation was statistically significant for nearly every strength range
(Fig. 4A), even those in weak and negative connection strength ranges, suggesting a global origin of the LPFC GBC-gF effect rather than a localized contribution of a subset of connections. Note that weak connections are typically ignored in rs-fcMRI studies due to their low signal-to-noise ratio but that the GBC measure may be effectively boosting signal-to-noise of widespread effects by averaging connectivity values across voxels (similar to improved local signal-to-noise from spatial smoothing) (Mikl et al., 2008).

Expanding on this analysis, we sought to identify whether there were distinct functional systems underlying these LPFC connection strength ranges. We observed that the strong positive connections with LPFC occurred with regions primarily in the frontoparietal control network, while weak positive and negative connections were made primarily with sensory-motor regions. Strong negative LPFC connections were primarily with the default mode network, a set of regions that are metabolically active at rest but deactivated during cognitive control tasks (Raichle, 2010). We formalized these observations by defining these 3 systems based on independent criteria from probabilistic cytoarchitecture and meta-analysis results (Eickhoff et al., 2005; Yarkoni et al., 2011) and using cutoffs from the strength-range distinctions described above (Fig. 5A). We found that LPFC connectivity significantly predicted $\mathrm{gF}$ in each of the 3 systems (Fig. $5 B$ ), supporting the conclusion that the LPFC GBC-gF effect is truly global rather than originating from within-network (strong positive) connections alone.

Several follow-up analyses were conducted to test for alternative explanations of these results. First, we measured the acrossindividual SD for each strength range to see if the stronger individual-differences effects were due to greater variance in the weaker strength ranges. Incompatible with this possibility, the SD actually went up along with (both positive and negative) connectivity strength. Second, we counted the number of voxels contributing to each strength range. We reasoned that if the LPFC GBC-gF correlation were truly global in origin, the correlation should be higher when more voxels are included rather than when connections are stronger. Compatible with a global origin, the highest negative and positive strength ranges had very low voxel counts (due to strict thresholds for these high strengths) and were the only strength ranges not statistically significant. 

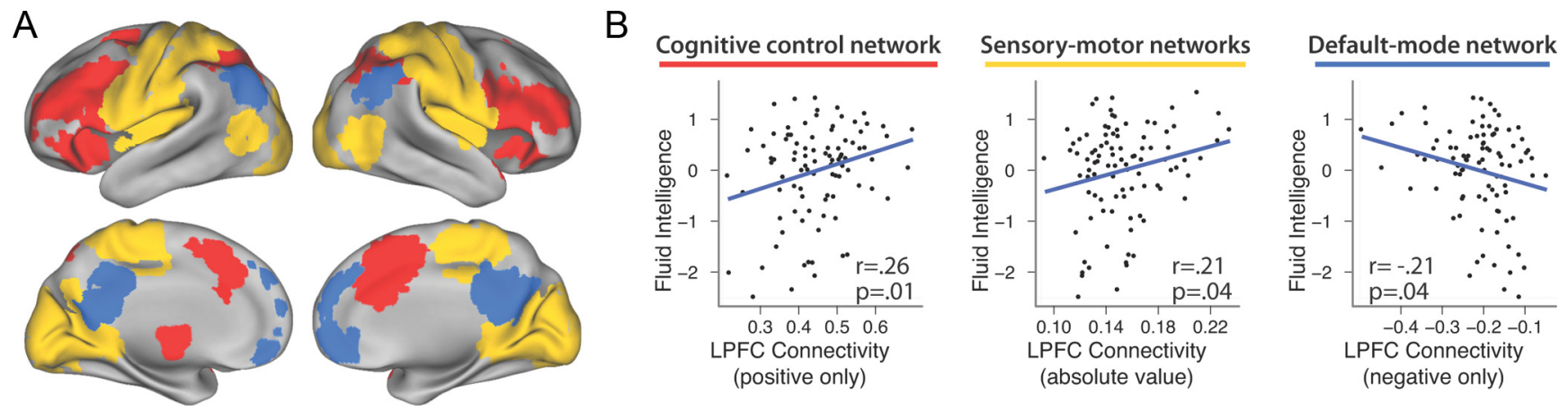

Figure 5. Testing for global or system-specific origins of the LPFC GBC-gF effect. $A$, We separated LPFC connectivity into 3 systems. The cognitive control network was identified using a meta-analysis of cognitive control studies, sensory-motor regions were identified based on probabilistic cytoarchitecture, and the default mode network was identified using a meta-analysis of studies investigating that network. $\boldsymbol{B}$, LPFC group average connectivity generally had high positive strength for cognitive control regions, low positive and negative strength (combined using absolute values) for sensory-motor regions, and high negative strength for default mode regions. We used the conjunctions between the masks in $A$ along with these group strength ranges to isolate the 3 systems. Individual differences in mean LPFC connectivity with each of the 3 systems correlated with $g F$, suggesting a truly global origin of the LPFC GBC $-g F$ effect.

As a final analysis, we tested whether the LPFC GBC- $\mathrm{gF}$ correlation was truly global in origin by examining whether the 3 functional systems identified in Figure 5 were correlated in terms of their predictive role in explaining gF variation. In other words, we hypothesized that the LPFC connectivity-gF correlation would not be statistically significant for any single functional system when accounting for individual differences in the other functional systems using multiple linear regression. Compatible with a global origin of the LPFC GBC-gF effect (in which individual differences in LPFC connectivity act in a unified fashion across systems), LPFC connectivity with each of the systems was no longer uniquely correlated with $\mathrm{gF}$ when the other 2 were included in a single linear model (all $p>0.21$ ). This was also true when each of the systems was modeled in pairs (control network + default network, control network + sensory-motor networks, or default network + sensory-motor networks; all $p>0.10)$. Further, individual differences in LPFC connectivity with the sensory-motor networks was correlated with the default mode network $(r=-0.56, p<0.001)$ and the cognitive control network $(r=0.69, p<0.001)$, while individual differences in LPFC connectivity with the cognitive control network was also correlated with the default mode network $(r=-0.59, p<0.001)$. The observation that gFcorrelating LPFC connectivity appears to vary between individuals as a whole, rather than in a system-specific manner, suggests that the contribution of LPFC connectivity to fluid intelligence can be best characterized in terms of global rather than connection-specific or system-specific connectivity.

\section{Estimating the relative effect size of the LPFC GBC-gF correlation}

The LPFC GBC-gF correlation effect size might be considered small ( $r=0.32,10 \%$ of the variance). To gain some perspective on this, we looked at the well known brain size-intelligence correlation (Willerman et al., 1991). Standard FreeSurfer gray matter segmentation (Dale et al., 1999) was used to estimate gray matter volume for each participant. Gray matter volume predicted gF with a correlation of $r=0.26$ ( $p=0.009 ; 6.7 \%$ of the variance). Critically, this is similar to the effect size of the LPFC GBC $-\mathrm{gF}$ correlation, $r=0.32$. In addition, the LPFC GBC-gF correlation explained independent variance $(r=-0.019$ between brain size and LPFC GBC), suggesting the LPFC GBC-gF is reflecting a unique mechanism underlying intelligence.
Also addressing the concern regarding effect size, we examined the LPFC activity-gF correlation, reported previously by Burgess et al. (2011). This effect size was smaller $(r=0.23, p=$ $0.03,5 \%$ of the variance) than the GBC-gF correlation in the same voxels $(r=0.30, p=0.004)$, again suggesting the LPFC $\mathrm{GBC}-\mathrm{gF}$ effect size is meaningful in relation to previous findings of fundamental factors underlying intelligence. Critically, the LPFC GBC-gF correlation again explained independent variance $(r=-0.05)$. This strongly suggests that the GBC effect is based on an independent mechanism supporting intelligence. Further, the lack of correlation between LPFC activity and LPFC GBC supports the conclusion that task activity was unlikely to have affected the GBC result.

Given the statistical independence of these 3 factors correlating with $\mathrm{gF}$, we built a single statistical model to see how much $\mathrm{gF}$ variance the 3 factors could explain together. We included only the 88 participants overlapping between the GBC and Burgess et al. (2011) analyses. Note that the whole brain gray matter volume correlation with $\mathrm{gF}$ was higher in these 88 individuals than the full set of 94 individuals $(r=0.33, p=0.0017)$. A linear model including LPFC GBC, LPFC lure activity, and whole brain gray matter volume was able to explain $26 \%$ of the variance in $\mathrm{gF}$ ( $r=$ $0.51, p=0.00001)$. All 3 factors significantly contributed to the model ( $p=0.001$ for LPFC GBC, $p=0.016$ for LPFC activity, and $p=0.0009$ for gray matter volume).

\section{Discussion}

We hypothesized, based on previous work demonstrating that control network regions have high GBC (Cole et al., 2010b), that the global connectivity of some control network regions would predict intelligence. Supporting this hypothesis, we found that left LPFC GBC predicted gF across individuals. Surprisingly, this effect was relatively specific to the left LPFC as only this region was statistically significant in a whole brain search. Further, this effect was statistically larger than in a variety of other regions and remained significant even after removing variance from other control network regions.

\section{Methodological implications}

Given that GBC is computed by averaging connection strengths, it is possible for a region's GBC to simply reflect a small set of strong connections (the region's "community") (Power et al., 2011). However, the present results demonstrate that a region's 
GBC can reflect connectivity throughout the brain and that it is possible for behavior connectivity relationships to be well characterized in terms of global connectivity.

Though weak connections have typically been ignored in rsfcMRI studies (Fox et al., 2005), the present results suggest (based on an independent behavioral measure) that these connections may be functionally relevant (Fig. 4). Rather than reflecting direct connectivity, these weaker connections might reflect distant indirect connections (Krienen and Buckner, 2009) between LPFC and other brain regions. Importantly, this suggests GBC with rs-fcMRI may be a particularly comprehensive way to assess functionally relevant connections, since this approach can detect connections even if functional relevance might be the result of long, multisynaptic chains. Alternatively, this weak connectivity could be the result of mixtures of strong positive and strong negative connectivity at a finer spatial grain than our multimillimeter voxels. Supporting this possibility, LPFC anatomical connections are known to project (directly, and indirectly via thalamus) to both excitatory and inhibitory neurons in macaque monkey sensory-motor cortices (Barbas and Zikopoulos, 2007).

We used whole brain signal regression as an rs-fcMRI preprocessing step. Recently, it has been argued that this might artifactually introduce rs-fcMRI negative connectivity by shifting the connectivity distribution (Murphy et al., 2009). However, others have demonstrated that connectivity is more functionally specific when using whole brain signal regression (Fox et al., 2009). The apparent utility of whole brain signal regression in conjunction with the remaining controversy regarding negative connectivity led us to use this method but to focus on positive connections in the main GBC-gF analysis (Fig. 2).

Importantly, however, our use of individual difference correlations with an independent behavioral measure could provide evidence regarding the functional relevance of negative connectivity with rs-fcMRI. We found that even negative LPFC connectivity correlated with gF. Critically, we would expect a positive correlation between negative connectivity and $\mathrm{gF}$ if negative resting state connectivity was actually positive connectivity shifted into negative connectivity ranges. In contrast to this prediction, the LPFC GBC-gF correlation involving negatively signed connections was also negative. In other words, when negative connections between LPFC and other brain regions were greater in sign (i.e., stronger), fluid intelligence was higher. This effect is the mirrored pattern of that observed with positive connections. Such an effect provides new evidence, based on an independent behavioral measure, that the strength of negative connections is not purely a measurement error but may also be functionally relevant.

\section{Limitations}

Previous research suggests that there are only minor differences between functional connectivity estimates from extended rest periods and concatenated short rest periods as used here (Fair et al., 2007). It remains possible, however, that some gF-correlating connectivity was altered by individuals' (task-related or taskindependent) mental states, especially given that task blocks were interspersed in time with the resting periods (Waites et al., 2005; Barnes et al., 2009). This possibility appears unlikely given that the GBC-gF correlation was not significant for a variety of regions with gF-correlating activity, and LPFC GBC did not correlate with LPFC activity $(r=-0.05)$. Even if mental state did affect the GBC-gF correlations, however, this would not invalidate the present results. Rather, it would shift our interpretation, suggesting that high gF individuals transiently increase their LPFC GBC more than low gF individuals. This would continue to suggest a key role for LPFC global connectivity in cognitive control but would indicate a different mechanism by which these brainbased and psychological factors are related. Future research into this possibility will be important for determining whether global connectivity - a region's integration with the entire brain-is relatively static or is changeable on a moment-to-moment basis.

Most previous studies that have investigated the relationship between connectivity and intelligence focused on specific connections (Jung and Haier, 2007; Song et al., 2008), local/withinnetwork integration (Li et al., 2009; Song et al., 2009), or overall global connectivity ( $\mathrm{Li}$ et al., 2009; Zalesky et al., 2011) rather than region-specific global connectivity. To our knowledge, only 2 studies have investigated the relationship between regionspecific global connectivity and intelligence: Langer et al. (2011) and van den Heuvel et al. (2009b; see their voxelwise secondary analysis). Our study represents a significant methodological and conceptual advance over this previous work. In contrast to Langer et al. (2011), we used fMRI to improve spatial localization relative to electroencephalography (Sharon et al., 2007), which is especially important for properly characterizing connectivity (Schoffelen and Gross, 2009). In contrast to van den Heuvel et al. (2009b), we used a large sample size $(N=94)$, corrected for multiple comparisons, and defined ROIs based on independent and theoretically meaningful functional criteria, thus avoiding circularity concerns (Kriegeskorte et al., 2009). It has been argued that using functionally defined regions is superior to using anatomically or arbitrarily defined regions for connectivity analysis since inadvertently including 2 regions in a single node can be detrimental to characterizing a graph (Smith et al., 2010; Wig et al., 2011). Further, the use of large sample sizes is especially critical when identifying individual differences effects, as recent methodological analyses have made it clear that studies with smaller sample sizes are likely subject to effect size inflation (Yarkoni, 2009), whereas studies with larger samples are likely to result in effect sizes closer to the true population effect (Braver et al., 2010). The results here demonstrate that these advances enabled significantly greater specificity in the functional inference drawn from the data; that is, the 3-way relationship between cognitive control, global connectivity, and fluid intelligence was only observed within a single brain region, the left LPFC.

Note that though our observed effect size $(r=0.32)$ was moderate, we would not expect any single fundamental factor to predict intelligence much more than this, given the many factors that likely contribute to intelligence. Supporting this conclusion, we found that 2 previously reported factors contributing to $\mathrm{gF}$ have equivalent effect sizes, brain size $(r=0.26)$ and LPFC activity $(r=0.23)$. Further, it may be that few brain behavior relationships are actually greater than this effect size, given that much of the existing literature reflects inflated effect sizes due to small sample sizes (see above) and/or double-dipping (Vul et al., 2009), which we avoided.

The present results might seem to suggest left LPFC is the "seat of intelligence." Critically, however, our analyses suggest LPFC is mechanistically unique rather than unique in its support of intelligence. Indeed, analyses with the same dataset indicate activity in a variety of regions support intelligence (Burgess et al., 2011; Preusse, 2011), while other studies show brainwide connectivity (van den Heuvel et al., 2009b), specific connections (Jung and Haier, 2007), and dopamine signaling (Schlagenhauf et al., 2012) also support intelligence. Our results provide novel evidence that left LPFC supports cognitive control and intelligence via a unique mechanism involving extensive global connectivity. Thus, it was 
critical that we rigorously tested for region-specific global connectivity rather than overall global connectivity (as most studies looking for intelligence correlations with global connectivity have done). One important possibility for future research to investigate is whether other control network regions are able to exert control over a variety of task-relevant regions via their influence on LPFC. Further, it will be important to assess whether other measures of connectivity might be more sensitive/accurate with respect to either global hub properties or unique mechanisms present in other cognitive control regions.

\section{Theoretical implications and future directions for research}

In contrast to studies emphasizing whole-brain network contributions to intelligence or studies emphasizing the contributions of specific regions (or networks) to intelligence, we found that a specific region's global connectivity predicts intelligence. This suggests a reconceptualization of LPFC as a functional hub that uses its brainwide influence to facilitate cognitive control and intelligence. Importantly, the observation of other cognitive control regions with high GBC but significantly lower GBC-gF correlations suggests that LPFC likely has some additional properties beyond extensive connectivity that underlie the observed GBC-gF correlation.

One possibility, based on Miller and Cohen's (2001) model of LPFC function, is that LPFC acts as a "flexible" hub, able to flexibly shift its connectivity with a variety of task-relevant regions according to task demands. Within this conceptualization, resting state fMRI GBC likely reflects the number of possible routes that LPFC can use during tasks to reconfigure connectivity. Thus, individuals with greater LPFC GBC would likely be able to dynamically reconfigure to a more specific connectivity pattern for any of a wide variety of possible task demands, increasing the specificity of LPFC's control over activity in those regions. This increased capacity for control across individuals would likely be reflected in the LPFC GBC-gF correlation. Further research is necessary to verify other predictions of the flexible hub hypothesis, such as task-dependent shifts in connectivity [but see Rowe et al. (2005), Cole et al. (2010a), Stelzel et al. (2011), and Deserno et al. (2012)].

We found that the LPFC GBC-gF correlation was present across a wide variety of strength ranges and spatial locations. Supporting the flexible hub account, many of these connections were with sensory and motor regions. This may reflect a particular architecture for a flexible neural system underlying human intelligence: Engineers have characterized flexible, adaptive control in terms of the ability for a system to access (both monitor and influence) multiple sources of task-relevant information (Åström and Murray, 2008). Additionally, although the functional importance of negative connectivity is not well understood (Murphy et al., 2009), the predictive relationship between $\mathrm{gF}$ and LPFC connections with the default mode network is consistent with a key role for inhibition of task-irrelevant processing in cognitive control and fluid intelligence (Anticevic et al., 2010). Further investigation into the functional relevance of cognitive control hubs, including the possibility that LPFC benefits from high global connectivity due to its unique flexibility, will be essential for understanding the neural architecture underlying human intelligence.

\section{References}

Anticevic A, Repovs G, Shulman GL, Barch DM (2010) When less is more: TPJ and default network deactivation during encoding predicts working memory performance. Neuroimage 49:2638-2648.
Åström KJ, Murray RM (2008) Feedback systems: an introduction for scientists and engineers. Princeton, NJ: Princeton UP.

Barbas H, Zikopoulos B (2007) The prefrontal cortex and flexible behavior. Neuroscientist 13:532-545.

Barnes A, Bullmore ET, Suckling J (2009) Endogenous human brain dynamics recover slowly following cognitive effort. PLoS One 4:e6626.

Braver TS, Cohen JD, Nystrom LE, Jonides J, Smith EE, Noll DC (1997) A parametric study of prefrontal cortex involvement in human working memory. Neuroimage 5:49-62.

Braver TS, Barch DM, Kelley WM, Buckner RL, Cohen NJ, Miezin FM, Snyder AZ, Ollinger JM, Akbudak E, Conturo TE, Petersen SE (2001) Direct comparison of prefrontal cortex regions engaged by working and longterm memory tasks. Neuroimage 14:48-59.

Braver TS, Cole MW, Yarkoni T (2010) Vive les differences! Individual variation in neural mechanisms of executive control. Curr Opin Neurobiol 20:242-250.

Burgess GC, Gray JR, Conway AR, Braver TS (2011) Neural mechanisms of interference control underlie the relationship between fluid intelligence and working memory span. J Exp Psychol Gen 140:674-692.

Cattell RB, Cattell AKS, Institute for Personality and Ability Testing (1973) Measuring intelligence with the Culture Fair Tests. Champaign, IL: Institute for Personality and Ability Testing.

Cole MW, Schneider W (2007) The cognitive control network: integrated cortical regions with dissociable functions. Neuroimage 37:343-360.

Cole MW, Bagic A, Kass R, Schneider W (2010a) Prefrontal dynamics underlying rapid instructed task learning reverse with practice. J Neurosci 30:14245-14254.

Cole MW, Pathak S, Schneider W (2010b) Identifying the brain's most globally connected regions. Neuroimage 49:3132-3148.

Cole MW, Anticevic A, Repovs G, Barch D (2011) Variable global diysconnectivity and individual differences in schizophrenia. Biol Psychiatry 70:43-50.

Conway AR, Engle RW (1996) Individual differences in working memory capacity: more evidence for a general capacity theory. Memory 4:577-590.

Conway RA, Cowan N, Bunting MF, Therriault DJ, Minkoff SRB (2002) A latent variable analysis of working memory capacity, short-term memory capacity, processing speed, and general fluid intelligence. Intelligence $30: 163-183$

Cox RW (1996) AFNI: software for analysis and visualization of functional magnetic resonance neuroimages. Comput Biomed Res 29:162-173.

Dale AM, Fischl B, Sereno MI (1999) Cortical surface-based analysis, I: segmentation and surface reconstruction. Neuroimage 9:179-194.

Dehaene S, Kerszberg M, Changeux JP (1998) A neuronal model of a global workspace in effortful cognitive tasks. Proc Natl Acad Sci U S A 95:14529-14534.

Deserno L, Sterzer P, Wüstenberg T, Heinz A, Schlagenhauf F (2012) Reduced prefrontal-parietal effective connectivity and working memory deficits in schizophrenia. J Neurosci 32:12-20.

DeYoung CG, Shamosh NA, Green AE, Braver TS, Gray JR (2009) Intellect as distinct from Openness: differences revealed by fMRI of working memory. J Pers Soc Psychol 97:883-892.

DeYoung CG, Hirsh JB, Shane MS, Papademetris X, Rajeevan N, Gray JR (2010) Testing predictions from personality neuroscience: brain structure and the big five. Psychol Sci 21:820-828.

Dosenbach NU, Visscher KM, Palmer ED, Miezin FM, Wenger KK, Kang HC, Burgund ED, Grimes AL, Schlaggar BL, Petersen SE (2006) A core system for the implementation of task sets. Neuron 50:799-812.

Duncan J, Seitz RJ, Kolodny J, Bor D, Herzog H, Ahmed A, Newell FN, Emslie H (2000) A neural basis for general intelligence. Science 289:457-460.

Duncan J (2010) The multiple-demand (MD) system of the primate brain: mental programs for intelligent behaviour. Trends Cognitive Sci 14:172-179.

Eickhoff SB, Stephan KE, Mohlberg H, Grefkes C, Fink GR, Amunts K, Zilles K (2005) A new SPM toolbox for combining probabilistic cytoarchitectonic maps and functional imaging data. Neuroimage 25:1325-1335.

Engle RW, Tuholski SW, Laughlin JE, Conway AR (1999) Working memory, short-term memory, and general fluid intelligence: a latent-variable approach. J Exp Psychol Gen 128:309-331.

Fair DA, Schlaggar BL, Cohen AL, Miezin FM, Dosenbach NU, Wenger KK, Fox MD, Snyder AA, Raichle ME, Petersen SE (2007) A method for using blocked and event-related fMRI data to study "resting state" functional connectivity. Neuroimage 35:396-405. 
Fales CL, Barch DM, Burgess GC, Schaefer A, Mennin DS, Gray JR, Braver TS (2008) Anxiety and cognitive efficiency: differential modulation of transient and sustained neural activity during a working memory task. Cognitive Affective Behav Neurosci 8:239-253.

Fischl B, Salat DH, Busa E, Albert M, Dieterich M, Haselgrove C, van der Kouwe A, Killiany R, Kennedy D, Klaveness S, Montillo A, Makris N, Rosen B, Dale AM (2002) Whole brain segmentation: automated labeling of neuroanatomical structures in the human brain. Neuron 33:341-355.

Fornito A, Zalesky A, Bassett DS, Meunier D, Ellison-Wright I, Yücel M, Wood SJ, Shaw K, O'Connor J, Nertney D, Mowry BJ, Pantelis C, Bullmore ET (2011) Genetic influences on cost-efficient organization of human cortical functional networks. J Neurosci 31:3261-3270.

Fox MD, Snyder AZ, Vincent JL, Corbetta M, Van Essen DC, Raichle ME (2005) The human brain is intrinsically organized into dynamic, anticorrelated functional networks. Proc Natl Acad Sci U S A 102:9673-9678.

Fox MD, Zhang D, Snyder AZ, Raichle ME (2009) The global signal and observed anticorrelated resting state brain networks. J Neurophysiol 101:3270-3283.

Friston KJ, Holmes AP, Worsley KJ, Poline JB, Frith CD, Frackowiak RSJ (1995) Statistical parametric maps in functional imaging: a general linear approach. Hum Brain Mapp 2:189-210.

Gottfredson L, Saklofske DH (2009) Intelligence: foundations and issues in assessment. Can Psychol 50:183-195.

Gottfredson LS (1997) Why g matters: the complexity of everyday life. Intelligence 24:79-132.

Gottfredson LS (2002) g: Highly general and highly practical. In: The general factor of intelligence: how general is it? (Sternberg RJ, Grigorenko, EL, eds), pp 331-380. Mahwah, NJ: Erlbaum.

Gray JR, Braver TS (2002) Personality predicts working-memory-related activation in the caudal anterior cingulate cortex. Cogn Affect Behav Neurosci 2:64-75.

Gray JR, Braver TS, Raichle ME (2002) Integration of emotion and cognition in the lateral prefrontal cortex. Proc Natl Acad Sci U S A 99:4115-4120.

Gray JR, Chabris CF, Braver TS (2003) Neural mechanisms of general fluid intelligence. Nat Neurosci 6:316-322.

Gray JR, Burgess GC, Schaefer A, Yarkoni T, Larsen RJ, Braver TS (2005) Affective personality differences in neural processing efficiency confirmed using fMRI. Cogn Affect Behav Neurosci 5:182-190.

Honey CJ, Sporns O, Cammoun L, Gigandet X, Thiran JP, Meuli R, Hagmann $P$ (2009) Predicting human resting state functional connectivity from structural connectivity. Proc Natl Acad Sci U S A 106:2035-2040.

Jo HJ, Saad ZS, Simmons WK, Milbury LA, Cox RW (2010) Mapping sources of correlation in resting state FMRI, with artifact detection and removal. Neuroimage 52:571-582.

Jung RE, Haier RJ (2007) The parieto-frontal integration theory (P-FIT) of intelligence: converging neuroimaging evidence. Behav Brain Sci 30:135154, discussion 154-187.

Kane MJ, Conway AR, Miura TK, Colflesh GJ (2007) Working memory, attention control, and the n-back task: a question of construct validity. J Exp Psychol Learn Mem Cogn 33:615-622.

Kriegeskorte N, Simmons WK, Bellgowan PSF, Baker CI (2009) Circular analysis in systems neuroscience: the dangers of double dipping. Nat Neurosci 12:535-540.

Krienen FM, Buckner RL (2009) Segregated fronto-cerebellar circuits revealed by intrinsic functional connectivity. Cereb Cortex:19:2485-2497.

Langer N, Pedroni A, Gianotti LRR, Hänggi J, Knoch D, Jäncke L (2012) Functional brain network efficiency predicts intelligence. Hum Brain Mapp 33:1393-1406

Lewis CM, Baldassarre A, Committeri G, Romani GL, Corbetta M (2009) Learning sculpts the spontaneous activity of the resting human brain. Proc Natl Acad Sci U S A 106:17558-17563.

Li Y, Liu Y, Li J, Qin W, Li K, Yu C, Jiang T (2009) Brain anatomical network and intelligence. PLoS Comput Biol 5:e1000395.

Lynall ME, Bassett DS, Kerwin R, McKenna PJ, Kitzbichler M, Muller U, Bullmore E (2010) Functional connectivity and brain networks in schizophrenia. J Neurosci 30:9477-9487.

Meng X, Rosenthal R, Rubin D (1992) Comparing correlated correlation coefficients. Psychol Bull 111:172-175.

Mikl M, Marecek R, Hlustík P, Pavlicová M, Drastich A, Chlebus P, Brázdil M,
Krupa P (2008) Effects of spatial smoothing on fMRI group inferences. Magn Res Imaging 26:490-503.

Miller EK, Cohen JD (2001) An integrative theory of prefrontal cortex function. Annu Rev Neurosci 24:167-202.

Murphy K, Birn RM, Handwerker DA, Jones TB, Bandettini PA (2009) The impact of global signal regression on resting state correlations: Are anticorrelated networks introduced? Neuroimage 44:893-905.

Nichols T, Hayasaka S (2003) Controlling the familywise error rate in functional neuroimaging: a comparative review. Stat Methods Med Res 12:419-446.

Nichols T, Brett M, Andersson J, Wager T, Poline JB (2005) Valid conjunction inference with the minimum statistic. Neuroimage 25:653-660.

Power JD, Cohen AL, Nelson SM, Wig GS, Barnes KA, Church JA, Vogel AC, Laumann TO, Miezin FM, Schlaggar BL, Petersen SE (2011) Functional network organization of the human brain. Neuron 72:665-678.

Power JD, Barnes KA, Snyder AZ, Schlaggar BL, Petersen SE (2012) Spurious but systematic correlations in functional connectivity MRI networks arise from subject motion. Neuroimage 59:2142-2154.

Preusse F (2011) Fluid intelligence allows flexible recruitment of the parieto-frontal network in analogical reasoning 5:22.

R Development Core Team (2009) R: a language and environment for statistical computing. Vienna: R Foundation for Statistical Computing.

Raichle ME (2010) Two views of brain function. Trends Cognitive Sci 14:180-190.

Raven J, Raven JC, Court JH (1996) Manual for Raven's progressive matrices and vocabulary scales. Oxford: Oxford Psychologists.

Rowe JB, Stephan KE, Friston K, Frackowiak RSJ, Passingham RE (2005) The prefrontal cortex shows context-specific changes in effective connectivity to motor or visual cortex during the selection of action or colour. Cereb Cortex 15:85-95.

Rubinov M, Sporns O (2011) Weight-conserving characterization of complex functional brain networks. Neuroimage 56:2068-2079.

Schaefer A, Braver TS, Reynolds JR, Burgess GC, Yarkoni T, Gray JR (2006) Individual differences in amygdala activity predict response speed during working memory. J Neurosci 26:10120-10128.

Schlagenhauf F, Rapp MA, Huys QJM, Beck A, Wüstenberg T, Deserno L, Buchholz HG, Kalbitzer J, Buchert R, Bauer M (2012) Ventral striatal prediction error signaling is associated with dopamine synthesis capacity and fluid intelligence. Hum Brain Mapp. Advance online publication. Retrieved May 29, 2012. doi:10.1002/hbm.22000.

Schoffelen J-M, Gross J (2009) Source connectivity analysis with MEG and EEG. Hum Brain Mapp 6:1857-1865.

Shallice T, Burgess PW (1991) Deficits in strategy application following frontal lobe damage in man. Brain 114:727-741.

Shamosh NA, DeYoung CG, Green AE, Reis DL, Johnson MR, Conway AR, Engle RW, Braver TS, Gray JR (2008) Individual differences in delay discounting: relation to intelligence, working memory, and anterior prefrontal cortex. Psychol Sci 19:904-911.

Sharon D, Hämäläinen MS, Tootell RB, Halgren E, Belliveau JW (2007) The advantage of combining MEG and EEG: comparison to fMRI in focally stimulated visual cortex. Neuroimage 36:1225-1235.

Shehzad Z, Kelly AM, Reiss PT, Gee DG, Gotimer K, Uddin LQ, Lee SH, Margulies DS, Roy AK, Biswal BB, Petkova E, Castellanos FX, Milham MP (2009) The resting brain: unconstrained yet reliable. Cereb Cortex 19:2209-2229.

Smith SM, Fox PT, Miller KL, Glahn DC, Fox PM, Mackay CE, Filippini N, Watkins KE, Toro R, Laird AR, Beckmann CF (2009) Correspondence of the brain's functional architecture during activation and rest. Proc Natl Acad Sci U S A 106:13040-13045.

Smith SM, Miller KL, Salimi-Khorshidi G, Webster M, Beckmann CF, Nichols TE, Ramsey JD, Woolrich MW (2010) Network modelling methods for FMRI. Neuroimage 43:1-17.

Song M, Zhou Y, Li J, Liu Y, Tian L, Yu C, Jiang T (2008) Brain spontaneous functional connectivity and intelligence. Neuroimage 41:1168-1176.

Song M, Liu Y, Zhou Y, Wang K, Yu C, Jiang T (2009) Default network and intelligence difference. Conf Proc IEEE Eng Med Biol Soc 2009:2212-2215.

Stelzel C, Basten U, Fiebach CJ (2011) Functional connectivity separates switching operations in the posterior lateral frontal cortex. J Cogn Neurosci 23:3529-3539.

Talairach J, Tournoux P (1988) Co-planar stereotaxic atlas of the human 
brain: 3-dimensional proportional system: an approach to cerebral imaging. Stuttgart: Thieme.

van den Heuvel MP, Mandl RC, Kahn RS, Hulshoff Pol HE (2009a) Functionally linked resting state networks reflect the underlying structural connectivity architecture of the human brain. Hum Brain Mapp 30:3127-3141.

van den Heuvel MP, Stam CJ, Kahn RS, Hulshoff Pol HE (2009b) Efficiency of functional brain networks and intellectual performance. J Neurosci 29:7619-7624.

Van Dijk KR, Hedden T, Venkataraman A, Evans KC, Lazar SW, Buckner RL (2010) Intrinsic functional connectivity as a tool for human connectomics: theory, properties, and optimization. J Neurophysiol 103:297-321.

Van Essen DC, Drury HA, Dickson J, Harwell J, Hanlon D, Anderson CH (2001) An integrated software suite for surface-based analyses of cerebral cortex. J Am Med Inform Assoc 8:443-459.

Van Essen DC (2005) A Population-Average, Landmark- and SurfaceBased (PALS) atlas of human cerebral cortex. Neuroimage 28:635-662.

Vincent JL, Patel GH, Fox MD, Snyder AZ, Baker JT, Van Essen DC, Zempel JM, Snyder LH, Corbetta M, Raichle ME (2007) Intrinsic functional architecture in the anaesthetized monkey brain. Nature 447:83-86.

Visscher KM, Miezin FM, Kelly JE, Buckner RL, Donaldson DI, McAvoy MP, Bhalodia VM, Petersen SE (2003) Mixed blocked/event-related designs separate transient and sustained activity in fMRI. Neuroimage 19:1694-1708.

Vul E, Harris C, Winkielman P, Pashler H (2009) Puzzlingly high correlations in fmri studies of emotion, personality, and social cognition 1. Perspect Psychol Sci 4:274-290.
Waites AB, Stanislavsky A, Abbott DF, Jackson GD (2005) Effect of prior cognitive state on resting state networks measured with functional connectivity. Hum Brain Mapp 24:59-68.

Wechsler D (2005) Wechsler Adult Intelligence Scale-III (WAIS-III). San Antonio: Pearson.

Wig GS, Schlaggar BL, Petersen SE (2011) Concepts and principles in the analysis of brain networks. Ann N Y Acad Sci 1224:126-146.

Willerman L, Schultz R, Neal Rutledge J, Bigler ED (1991) In vivo brain size and intelligence. Intelligence 15:223-228.

Yarkoni T (2009) Big correlations in little studies. Perspect Psychol Stud 4:294-298.

Yarkoni T, Barch DM, Gray JR, Conturo TE, Braver TS (2009) BOLD correlates of trial-by-trial reaction time variability in gray and white matter: a multi-study fMRI analysis. PLoS One 4:e4257.

Yarkoni T, Braver T, Szymura E (2010) Cognitive neuroscience approaches to individual differences in working memory and executive control: conceptual and methodological issues. Handbook of individual differences in cognition: Attention, memory and executive control. New York: Springer.

Yarkoni T, Poldrack RA, Nichols TE, Van Essen DC, Wager TD (2011) Large-scale automated synthesis of human functional neuroimaging data. Nat Methods 8:665-670.

Zalesky A, Fornito A, Seal ML, Cocchi L, Westin CF, Bullmore ET, Egan GF, Pantelis C (2011) Disrupted axonal fiber connectivity in schizophrenia. Biol Psychol 69:80-89. 